dann in einen Sammelschacht abgeführt wird. Alle zwei bis drei Wochen werden die Verteilungsrinnen aufgeharkt und etwa alle sechs Monate erneuert.

Die Aufnahmefähigkeit der Füllkörper beträgt nach längerem Betriebe etwa $30 \%$ und kann die durchschnittliche Menge des auf $1 \mathrm{cbm}$ Filtermaterial gereinigten Abwassers bei den einstufigen Filtern auf $1 \mathrm{cbm}$. Abwasser, bei den zweistufigen auf $0,66 \mathrm{cbm}$, bei den Tropfkörpern auf $1 \frac{1}{2} \mathrm{cbm}$ pro Tag berechnet werden.

Der Betrieb der Versuchsanlage, welche nunmehr fast $2^{1 / 2}$ Jahre besteht, wurde nur durch Hochfluten wenige Tage unterbrochen und durch mehr als 2000 Untersuchungen des Abwassers im Chemischen Untersuchungsamte der Stadt Aachen eingehend geprüft. Die Untersuchung erstreckte sich außer auf die qualitative Prüfung von Schwefel- wasserstoff, salpetriger Säure und. Salpetetsäurc, auch auf die quantitative Bestimmung des Ammoniakgehaltes, des Gehaltes an Gesamtstickstoff und organischem Stickstoff, sowie auf den Verbrauch an Kaliumpermanganat. In vielen Fällen wurden auch die Gesamtschwebestoffe, sowie die Menge der organischen und anorganischen Teile derselben bestimmt.

Das Rohwasser zeigte im Durchschnitt einen Gehalt von $800 \mathrm{mg}$ suspendierter Stoffe, während der Gehalt an löslichen Stoffen etwa $1200 \mathrm{mg}$ im Abwasser beträgt. Im Mittel von $68 \mathrm{im}$ Jahre 1908 ausgeführten Untersuchungen betrug der Gehalt des Rohwassers an Ammoniak $39,9 \mathrm{mg}$, an Gesamtstickstoff $61,7 \mathrm{mg}$, an organischem Stickstoff $27,2 \mathrm{mg}$; der Verbrauch an $\mathrm{KMnO}_{4} 217 \mathrm{mg}$ pro Liter. Dagegen betrug das Jahresmittel von

$\begin{array}{cccc}\text { an } \mathrm{NH}_{3} & \text { Ges. N, } & \text { Org. N, } & \text { KMnO }_{4} \\ 52,3 & 52,2 & 9,4 & 157,0 \\ 13,8 & 15,9 & 4,4 & 47,8 \\ 17,6 & 20,3 & 5,1 & 54,4 \\ 14,8 & 13,4 & 3,4 & 48,4 \\ 14,7 & 14,3 & 1,7 & 46,7 \\ 25,6 & 26,4 & 5,6 & 68,9\end{array}$

Der prozentige Reinigungseffekt obiger Filter berechnet sich hiernach bei dem Abwasser des

Faulraumes auf eine Zunahme . . . . . $31 \%$

Filters Va auf eine Abnahme . . . . . $66 \%$

Filters $\mathrm{Vb}$ auf eine Abnahme . . . . . $56 \%$

Tropffilters A Koks auf eine Abnahme . . $62 \%$

Tropffilters A. Kreien auf eine Abnahme . $65 \%$

Tropffilters B auf eine Abnahme... . . $37 \%$ $\underset{\text { Ges.N }}{\text { an }}$ Ges.N
von

Faulraumes auf eine Abnahme. . . . . . $17 \%$
Filters $\mathrm{Va}$ auf eine Abnahme . . . . . $75 \%$

Filters $\mathrm{Vb}$ auf eine Abnahme . . . . . . . $68 \%$

Tropffilters A. Koks auf eine Abnahme . 79\%

Tropffilters A. Kreien auf eine Abnahme . $77 \%$

Tropffilters B auf eine Abnahme... . . $57 \%$

an Org.

N. von

Faulraumes auf eine Abnahme . . . . $64,7 \%$

Filters Va auf eine Abnahme . . . . . $83,9 \%$

Filters Vb auf eine Abnahme . . . . 81,1\%

Tropffilters A. Koks auf eine Abnahme . $87,5 \%$

Tropffilters A. Kreien auf eine Abnahme 93,8\%

Tropffilters B auf eine Abnahme . . . 79,5\% $\underset{\text { von }}{\mathrm{KMnO}_{4}}$

Faulraumes auf eine Abnahme . . . . $27,7 \%$ Filters Va auf eine Abnahme . . . . . 78,0\% Filters $\mathrm{Vb}$ auf eine Abnahme . . . . 75,0\% Tropffilters A. Koks auf eine Abnahme . $77,7 \%$ Tropffilters A. Kreien auf eine Abnahme $78,6 \%$ Tropffilters B auf eine Abnahme . . . . $68,3 \%$

Von den gereinigten Abwässern werden außer den für die chemische Untersuchung erforderlichen Proben täglich Proben entnommen, welche je in einer geschlossenen und einer offenen Flasche mindestens 10 Tage, teilweise monatelang aufbewahrt und auf FäuInisfähigkeit hin beobachtet werden.
Ausnahmslos haben sich die gereinigten Abwässer als nicht mehr fäulnisfähig erwiesen, und ihre $\mathrm{Oxy}$ dierbarkeit nahm nach kurzer oder längerer Aufbewahrung entsprechend ab. Die gereinigten Wässer insbesondere auch die der Tropffilter sind durchweg klar oder von höchstens schwach opaler Färbung, welche stets nach einem Tage verschwindet; sie sind geruchlos oder weisen höchstens einen schwach erdigen Geruch auf. Das Rohwasser wird in den Faulbecken sehr gut für die biologische Reinigung in den Oxydationsbecken vorbereitet, die suspendierten Stoffe desselben, von denen ca. $90 \%$ in den Faulbecken bleiben, erleiden dort eine Verminderung von ca. 50\% und liefern einen Schlamm, welcher leicht austrocknet, ohne einen unangenehmen Geruch zu verbreiten, ohne Belästigung für die Nachbarschaft gelagert werden kann und einen guten Dünger, insbesondere für Wiesen, bildet.

Die Tropffilter zeigen bei nahezu gleichem Reinigungserfolge eine um etwa $50 \%$ erhöhte Leistungsfähigkeit gegenüber den geschlossenen Füllkörpern, die Anlagekosten derselben, namentlich des Tropffilters B, sind wesentlich geringer, so daB die Ausführung dieser Filter aus wirtschaftlichen Gründen bei weitem vorzuziehen ist.

\section{Rechte der Angestellten und Arbeiter an den Erfindungen ihres Etablissements ${ }^{1}$ ).}

Von Rechtsanwalt Dr. H. JAcoBson.

(Eingeg. d. 25.11. 1907.)

Eine wissenschaftliche Arbeit, die auch der Andersdenkende mit Freuden begrüBt, und deren Vorzüge er bereitwillig anerkennt, muB zweifellos

1) Auf dem letzten Juristentage wurde die äuBerst interessante Frage erörtert, ob Erfindungen, 
ihren Wert haben. So ist es mit der kleinen Schrift des bekannten ehemaligen Senatspräsidenten am Reichsgericht, Bolze: ,Rechte der Angestellten und Arbeiter an den Erfindungen ihres Etablissements" der Fall, mit der ihr Verfasser zu einer der am hoißesten umstrittenen Fragen des letzten Juristentages Stellung nimmt.

Bisher hat der deutsche Gesetzgeber für diese Frage keine Lösung gefunden. Die wenigen Lösungen, die in anderen Staaten versucht worden sind, sind einander gerade entgegengesetzt. Österreich gewährt dem angestellten Arbeiter das Urheberrecht an den von ihm im Dienste gemachten Erfindungen und verpflichtet den Arbeitgeber zur entsprechenden Entschädigung, falls etwa im Vertrage anderes vereinbart worden sein sollte. Finnland gewährt das Urheberrecht an der Erfindung des Angestellten dessen Dienstherrn; auf dem gleichen Standpunkte stehen Ungarn und Dänemark, dieses wenigstens hinsichtlich des Staates als Arbeitgeber.

Die anderen Gesetzgeber sind dem Anscheine nach nicht zur Lösung gelangt. Doch bringt Bolze namentlich aus der englischen und französischen Rechtsprechung und Literatur eine Fülle von Stoff.

Die Schwierigkeit der Frage liegt in den mannigfach verschiedenen Verhältnissen, in denen Erfindungen zustande kommen.

Hier arbeitet ein vermögensloser, karg bezahlter Techniker von ungemein scharfer Beobachtungsgabe und sieghaft glänzendem Denken. Die täglichen Schwierigkeiten, die sich in seinem Dienste ergeben, drängen ihn zum Nachdenken; die Erfahrungen, die er in seinon Dienststellungen gesammelt, erleichtern ihm den Weg, und seinem Sinn drängt sich eine einfache Vorrichtung auf, die Hunderte von Händen erspart, Schutz vor stündlichen Gefahren bietet und so die Herstellung irgend eines Stoffes um die Hälfte verbilligt. Soll nun lediglich der Dienstherr den Gewinn von der Energie des Nachdenkens seines Angestellten haben, weil sich diesem zufällig in seinem Betriebe die Beobachtung aufdrängte?

Dort ist eine große Aktiengesellschaft, die jährlich Zehntausende für vielleicht vergebliche Versuche opfert, die ihre wissenschaftlich gebildeten Angestellten, wenn auch nicht reichlich, jedoch angemessen entschädigt. Ihre Leiter erkennen ein Problem, dessen Lösung von ungeheurem Werte nicht nur für dieses Unternehmen, sondern vielleicht für die ganze Menschheit ist. Nach jahrelangen, kostspieligen Versuchen wird schließlich ein glückliches Ergebnis erzielt, das ohne die planmäBigen Vorar-

welche Angestellte und Arbeiter in gewerblichen Etablissements machen, das Eigentum derselben bleiben, oder ob die Betriebsunternehmer über das Nutzungsrecht verfügen und insbesondere dazu berechtigt sind, Patente zu nehmen und Gebrauchsmuster anzumelden.

Der bekannte ehemalige Reichsgerichtssenatspräsident, Dr. Bolze, hat dieses Thema soeben in interessanter und fesselnder Weise in einer Broschüre behandelt, die im Verlage der A $\mathrm{k}$ a d e m is c h e $\mathrm{n}$ Verlagsgesellschaft m. b. H. in Le i p zig jüngst erschienen ist.

Diese Arbeit, welche nicht allein für Juristen, sondern auch für alle Fabrikanten und gewerblichen Unternehmer von größter Wichtigkeit ist, wird in den beteiligten Kreisen gewiß starkem Interesse begegnen. beiten sämtlicher Angestellten dieses Fachs bei aller Anerkennung der Tüchtigkeit und des Verdienstes dessen, dem schließlich die Lösung glückte, nicht erreichbar gewesen wäre. Soll nun etwa derjenige, der die Arbeit sozusagen schon im Reifestadium übernommen und glücklich zu Ende geführt hat, den Patentschutz für sich allejn erwerben und möglicherweise damit eine Konkurrenzfirma unterstützen können?

In einer anderen Fabrik stellen Arbeiter immer denselben Gegenstand auf dieselbe Art und Weise her. Durch die lange Ubung werden sie mit der Herstellungsmaschine besonders vertraut. Sie erleichtern sich schlieBlich die Arbeit, indem sie eine Vorrichtung an der Maschine anbringen, die die Herstellung vereinfacht, beeilt oder sie vor Gefahren bei der Herstellung schützt. Dabei ging es sehr einfach zu : Der eine brachte einen neuen Hebel an der Maschine an, wieder nach einiger Zeit gab ein anderer dem Hebel eine praktischere Form, schließlich beseitigte ein dritter noch eine Reibung, die den Gebrauch des Hebels vollends erleichterte. Vielleicht sind schon die beiden ersten Arbeiter längst in andere Fabriken gegangen, ohne zu ahnen, daB ihr zusammenhangloses und doch ineinandergreifendes Arbeiten die Vollendung einer Erfindung von großem Werte verursachte. Wem gehört in diesem Falle der Patentschutz?

Man sieht ohne weiteres, daß die Fälle jedesmal so verschieden sind, daß sie kaum unter einen recht. lichen Gesichtspunkt vereinigt werden können. Nur das eine haben sie gemein, daß sie sämtlich patentfähige Erfindungen darstellen. Aber auch nur hierüber, über die Patentfähigkeit und den Inhalt des Patents, gibt das Patentgesetz Aufschluß. Ohne zu fragen, wem die Ehre und die Vermögensvorteile der Erfindung gebühren, hat das Kaiserliche Patent. amt den Schutz demjenigen zu erteilen, der die Erfindung anmeldet. Aus der Novelle von 1891 hat der Anmeldende freilich keinen Anspruch auf Erteilung des Patents, ,wenn der wesentliche Inhalt seiner Anmeldung den Beschreibungen, Zeichnungen Modellen, Gerätschaften oder Einrichtungen eines anderen oder einem von diesem angewendeten Verfahren ohne Einwilligung desselben entnommen und von dem letzteren aus diesem Grunde Einspruch erhoben ist." Allein das Patentgesetz sagt nichts über den Fall, daß der Anmeldende zu der Entlehnung berechtigt war. Er hat z. B. das Modell nach seinen Anweisungen von einem Mechaniker anfertigen lassen. Nur über den Inhalt des Patentes läßt sich das Gesetz aus. Es gibt dem Patentinhaber die ausschlieBliche Befugnis zur gewerblichen Nutzung des Gegenstandes der Erfindung. Es durchbricht dieses sein Prinzip lediglich zugunsten desjenigen, der die Erfindung im Inlande bereits in Benutzung genommen, denn dieser darf sie auch für den eignen Betrieb weiter ausnutzen. Die Fälle, wo das allgemeine Wohl ein ausschließliches Einzelrecht verbietet, können hier unberücksichtigt bleiben.

Seiner ganzen Natur nach ist das Patentgesetz überhaupt nicht der Ort, wo sich der Gesetzgeber darüber aussprechen könnte, wem das Recht zu dem Patente zusteht. Wie aus dem vorher gesagten folgt, begnügt es sich mit der reinen Form der Anmeldung und gibt dem Anmelder die ausschließ- 
lichen Befugnisse des Patentes; mit den privatrechtlichen Verhältnissen zwischen dem etwaigen Erfinder und dritten Personen beschäftigt es sich überhaupt nicht. Wer wie B o l z e daher vom Patentgesetz ausgeht, schafft infolgedessen aus dem Nichts, während sich tatsächlich aus anderen Satzungen auch die Rechtssätze über das Erfinderrecht ableiten lassen, oder sich wenigstens auf anderen Rechts. gebieten bereits urbar gemachter Boden für künftige Aussaat befindet.

Daran kann uns nicht irre machen, daß in anderen Gesetzgebungen die Frage nach dem Recht der Ange tellten an der Erfindung in den Patentgesetzen erledigt ist. Bloß dem Nachahmungstriebe zu Liebe konstruktive Fehler anderer Staaten mitzumachen, haben wir keine Veranlassung. Fragen über Beziehungen einander im Rechtsleben nebengeordneter Personen - und dazu gehören auch die Beziehungen zwischen Geschäftsherren und Angestellten - gehören dem bürgerlichen Rechte an.

Nun wird in der Regel das Verhältnis zwischen Arbeitgeber und Angestellten eine Dienstmiete sein. Der Dienstvertrag überträgt jedoch nur Ansprüche aus einem Schuldverhältnis, niemals Eigentumsrechte, wobei es keinen Unterschied macht, ob das Eigentum an einem materiellen, einer Sache, oder einem immateriellen Gute, einem Rechte, entstehen würde. Laut dem Dienstvertrage stellt der Angestellte während einer bestimmten Zeit seine Arbeitskraft in den Dienst des Geschäftsherrn. Nun wird in den weitaus meisten Fällen gar nicht festzustellen sein, ob der Angestellte die Erfindung während der Geschäftszeit gemacht, oder ob er lediglich die Anregung dazu in der Geschäftszeit empfangen, sie aber in seinen Mußestunden ausgeführt hat. Auch aus $\$ 855$ B. G. B., wie jetzt einige wollen, läßt sich nicht folgern, daß der Angestellte nur Besitzdiener für seinen Herrn an dem erst entstehenden immateriellen Gute sei, das noch keine Sache und bis zum Momente der Patentierung auch noch nicht einmal ein Recht darstellt. Vor allen Dingen wird eben die Prinzipienfrage, ob die Erfindung im Betriebe des Erwerbsgeschäfts entstanden oder nicht, als gelöst bereits angesehen. Weder im Gesetz, noch beim Mangel anderweitiger Anhaltspunkte für den Willen der Parteien ist im Vertrage eine Grundlage dafür gegeben, daß der Angestellte mehr zu leisten habe als die Indienststellung - seiner Arbeitskraft. Diese auch von $\mathrm{E} \mathrm{dwin} \mathrm{Ka} \mathrm{z}$ im ,Gewerblichen Rechtsschutz" Bd. II, 3213ff. vertretene
Auffassung kann auch nicht durch dic mit unendlichem Fleiß von $\mathrm{B}$ ol ze zusammengetragene Literatur erschüttert werden. Gewiß macht sich für den Fall, wo der Geschäftsherr Anleitung und Ziel der Forschung angegeben hat, eine besondere Gesetzgebung nötig, obwohl gerade in diesen Fällen wohl regelmäBig der Anstellungsvertrag Bestimmungen über das Recht der Erfindung enthalten wird. Fraglos erscheint jedoch, daß die sogen. Etablissementserfindung, d. h. die durch die Erfindung mehrerer erzielte, dem Betriebe zuwächst. Selbst aber in den Fällen, in denen, sei es durch eine künftige Gesetzgebung, sei es durch ausdrücklichen Vertrag, die Erfindung dem Geschäftsherrn zuwächst, würde eine billige Gesetzgebung dem Erfinder einen Anspruch sowohl auf entsprechende Entschädigung als auch insbesondere das sehr wesentliche Namensrecht an der Erfindung zuerteilen. Denn der aus dem Kreise der Techniker immer wieder laut gewordene, gerade hierauf gerichtete $W$ unsch entspringt nicht etwa einer Erfindereitelkeit, sondern die Ehre einer Erfindung ist ein sehr wesentliches Vermögensrecht. Denn derjenige, der nachweisen kann, daß er mehr oder minder Erfindungen gemacht, wird leicht und zu besseren Bedingungen jederzeit in verschiedenen Betrieben eine neue Anstellung finden.

\section{Zur Abwehr.}

Von Dr. F. Raschig-Ludwigshafen a. Rh.

Auf Seite 794 dieser Zeitschrift sprechen Lunge und Berl von einer absichtlichen Verzerrung des Tatbestandes, die ich mir ihnen gegenüber hätte zu schulden kommen lassen, und die für meine Polemik charakteristisch sei.

Demgegenüber stelle ich nochmals als Tatbestand fest, daß schon von Tra u tz die Nitrosisulfosäure (Sulfonitronsäure von Lunge und Berl) als Zwischenprodukt des Bleikammerprosses nachgewiesen wurde, daß ich diesen Nachweis bestätigte und vertiefte, daß L unge und Berl (diese Z. 1906, 887/88) dies auch zugeben, daß aber trotzdem ein halbes Jahr später (diese Z. 1906, 1932, Zeile 34) Lunge wörtlich von, ,dieser von uns (Lunge und Berl) als solche nachgewiesenen Zwischenstufe* spricht.

\section{Referate.}

\section{I. Chemische Technologie (Apparate, Maschinen und Verfahren allgemeiner Verwendbarkeit).}

Wasserreiniger. (Z. f. Dampfkessel- u. Maschinenbetr. 29, 218 [1906].)

Nach Betrachtungen allgemeinerer Art über Kesselspeisewasserreinigung und Hinweisen auf die in dieser Richtung grundlegenden Arbeiten von $\mathrm{Clark}$ und Porter findet die von $\mathrm{Blach}$ er angegebene Art der schnellen Ermittlung der Ent. härtungszusätze speziellere Besprechung. Desgleichen der Schroedersche Wasserreinigungsapparat, der gegenüber anderen Konstruktionen sich insbesondere durch Fortfall eines größeren Filters unterscheidet. g.-

F. W. Foos. Die rationelle Kesselspeisung mit beson derer Berïcksichtigung der Brikettindustrie. (Braunkohle 5, 293 [1906].)

Verf. empfiehlt vor allem Reinigung des Speisewassers, sowohl von Kesselsteinbildnern, als besonders auch von mitgerissenem Schmieröl. Die üblichen 